\title{
PUBLISHER CORRECTION OPEN Publisher Correction: First-principles calculation of intrinsic defect chemistry and self-doping in PbTe
}

\author{
Anuj Goyal ${ }^{1,2}$, Prashun Gorai ${ }^{1,2}$, Eric S. Toberer ${ }^{1,2}$ and Vladan Stevanović ${ }^{1,2}$ \\ npj Computational Materials (2017)3:51 ; doi:10.1038/s41524-017-0054-7
}

Correction to: npj Computational Materials (2017); doi:10.1038/ s41524-017-0047-6; Published 10 October 2017

Figure 4 in the original version of this paper did not include shading to indicate band gap. This figure has now been corrected, in the HTML and PDF versions of this article.

Open Access This article is licensed under a Creative Commons Attribution 4.0 International License, which permits use, sharing, adaptation, distribution and reproduction in any medium or format, as long as you give appropriate credit to the original author(s) and the source, provide a link to the Creative Commons license, and indicate if changes were made. The images or other third party material in this article are included in the article's Creative Commons license, unless indicated otherwise in a credit line to the material. If material is not included in the article's Creative Commons license and your intended use is not permitted by statutory regulation or exceeds the permitted use, you will need to obtain permission directly from the copyright holder. To view a copy of this license, visit http://creativecommons. org/licenses/by/4.0/.

(c) The Author(s) 2017 
(a)

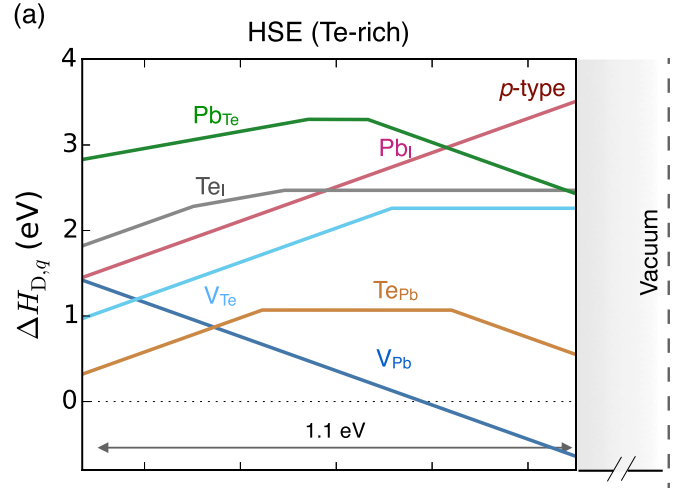

(c)

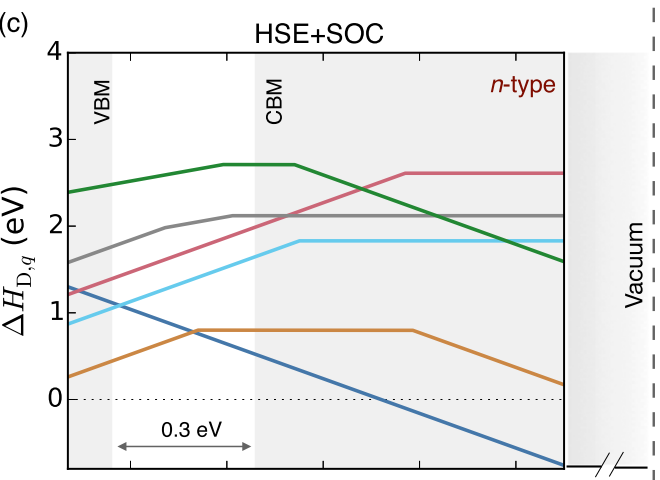

(e)

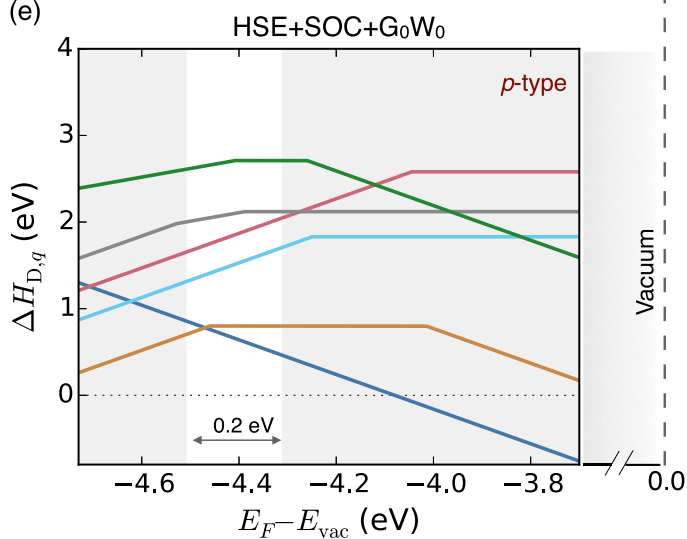

(b)

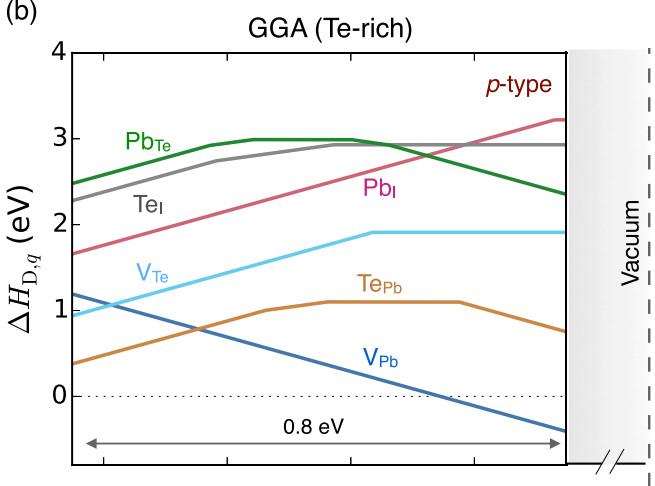

(d)

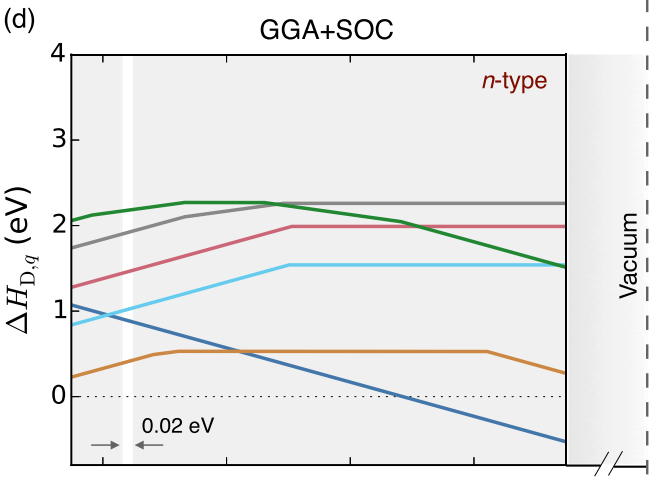

(f)

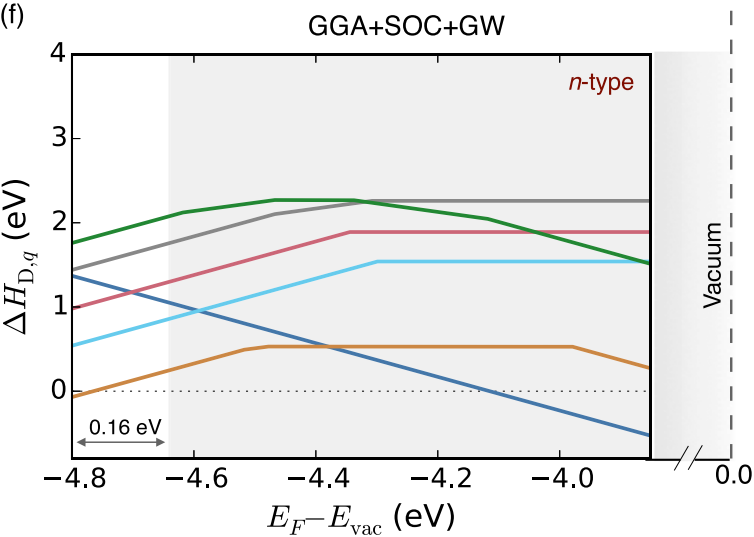

Fig. 4 Defect formation energy as a function of Fermi energy (referenced to the vacuum level) for native vacancies, anti-sites and interstitials in PbTe under Te-rich conditions. Calculated with a HSE, b GGA, c HSE with spin-orbit coupling (SOC), d GGA + SOC, e HSE + SOC with band edge shifts from single step $G_{0} W_{0}$, and $\mathbf{f} G G A+S O C$ with band edge shifts from self-consistent $G W$ calculations. Band edges (VBM and CBM) are aligned on the absolute scale with vacuum, and band gap is shown in the white region. Major differences in defect plots between different levels of theory comes from differences in the band edge positions 